\title{
Numerical characterization of the effects of flow rate on pressure and velocity distribution of pump as turbine
}

\author{
Yong Gao*, Xiaoyong Fan and Rui Dang \\ School of Chemistry and Chemical Engineering, Yulin University, Yulin 719000, China
}

\begin{abstract}
Small hydropower systems are the best solution for electromechanical components through the use of pump operating at inverse conditions as turbine for power generation in rural settlements of under developed countries especially since its construction and maintenance is cheap. This study investigates the effects of flow rate and internal flow characterization of a model centrifugal pump operating at a rotational speed of $n=1400 \mathrm{rpm}$ with 4 main blades and 4 splitters at a head of $18(\mathrm{~m})$ and flow of $12.5\left(\mathrm{~m}^{3} / \mathrm{h}\right)$. The experiment and numerical calculations were performed, and pressure and velocity were analysed based on the results obtained. According to the results obtained, velocity increased by movement of flow through the flow passage. As the discharge increased, head decreased but the power required to run the pump increased. Centrifugal pump can perform in reverse mode as a hydro turbine devoid of any modification on mechanical components with a maximum efficiency of $62.07 \%$ recorded at $Q=20 \mathrm{~m}^{3} / \mathrm{h}$. The study helps to recognize the numerical flow characterization and serves as a supporting document to provide vital information on centrifugal pumps to other studies.
\end{abstract}

Keywords: Hydraulic performance, pump as turbine, rotational speed, transient flow characteristics.

STUDIES on pump operating in inverse mode as a traditional turbine (PAT) have been carried out by many researchers since 1930, and the results show that PAT can be considered as a perfect alternative for power generation, due to its benefits ${ }^{1}$. Zhang et al. ${ }^{2}$ describe the need for small hydropower stations. Agarwal ${ }^{3}$ emphasized the advantages of using a micro-hydroelectric turbine PAT with more types of head and flow rates, availability of parts such as seals and bearings, and easier installation. Likewise, Williams ${ }^{4}$ emphasizes the economic advantages and easier operation of PAT. However, this solution is usually very broad and can not be easily implemented in existing urban networks. Choosing a commercial pump PAT $^{5}$ is not always an easy task because the pump manufacturer does not supply the

\footnotetext{
*For correspondence. (e-mail: gaoyong5407@163.com)
}

working conditions in reverse. Therefore, recent research on PAT was based on the development of the inverse model of the predictive model of centrifugal pumps ${ }^{6-8}$. These techniques are based primarily on geometric, theoretical and experimental analysis. PAT performance was investigated under different rotational speeds ${ }^{9,10}$ by the experimental study of a hydraulic system. The results show that the characteristics of PAT can be predicted from the pump characteristics and performance curves to a certain extent for the operation of the PAT at the same height and speed. These studies were conducted by Joshi et $a l .{ }^{11}$. In the case of hydroelectric micro-locations that generate $25 \mathrm{~kW}$ of electricity from a $5.5 \mathrm{~m}$ head, a simple method was used to predict the performance of the PAT to aid pump selection (specific high-speed pumps). Joshi et al. created a relationship between the specific speed of the pump and PAT mode to help choose PAT for a specific site of a hydropower station ${ }^{11}$. The performance of pump as turbine is directly dependent on the performance of the pump. Several studies have been conducted on the performance of pumps in pump and turbine modes ${ }^{10-13}$. Normally, pump as turbine efficiency point (BEP) is attained similarly as that of a pump at higher head and discharge $e^{10,14,15}$. As a result, it is expected that the hydraulic loss, flow instability in pump mode will be different from PAT. According to the analytical study, there is a greater axial thrust in the PAT mode than pump mode ${ }^{16}$. These parameters can have different effects on the process of energy conversion in PAT mode than pump at varied speeds. Therefore, it is necessary to study PAT performance at different speeds. Research on PAT performance at different rotational speeds are minimal for instance $^{10,15,17}$. Additionally, Zhang ${ }^{18}$ and Zhang et al. ${ }^{19,20}$ also investigated the energy conversion of low specific speed centrifugal pump and some other vortex pump. However, the results were not accurate to prove the performance. Hence this study analyses the flow rate effects to help provide information for future research in PAT.

\section{The experiment background}

Experiments were performed using an open test loop with a selected centrifugal pump model to perform as a 


\section{RESEARCH ARTICLES}

turbine. The pump mode experiment was conducted to validate CFD results. Power, efficiency, flow and head results obtained from the experiment was measured considering different flow conditions. Flow rate varies flanked by $Q=5(\mathrm{~m} 3 / \mathrm{h})$ as the minimum and $Q=25$ $(\mathrm{m} 3 / \mathrm{h})$ as the maximum. The diagram of the experimental arrangement is displayed in Figure 1.

\section{Results and discussions}

Analysis of hydroelectric centrifugal pump curves: The selected hydroelectric centrifugal pump performance results were attained at diverse flow conditions at two different rotational speeds. The flow adjustment was made to obtain the expected results. The discharge pressure suction and other important geometrical parameters and equipment were used and the results obtained were analysed in Figure 2. CFD was calculated to validate the results obtained by the experiment. The efficiency and head obtained numerically and experimentally were compared and analysed considering the respective flow rates. It is observed from the results that there is a slight difference between CFD and the experiment result. The head of the experimental result is marginally higher than the CFD, as the efficiency of the CFD after the design flow rate is slightly higher than the experimental efficiency. At the BEP, $Q=20\left(\mathrm{~m}^{3} / \mathrm{h} \%\right)$ at $n=1400 \mathrm{rpm}$. A deviation of around $2.8 \%$ was recorded while comparing the efficiency of numerical calculations and experiment results. The deviation recorded at the PUMP design point between experimental and numerical results for the head and efficiency were $5.3 \%$ and $8.9 \%$ at $n=1400 \mathrm{rpm}$ respectively. The highest deviation was recorded at the $Q_{\mathrm{BEP}}$ on the head curve which makes the simulation results dependable for further analyses.

\section{Unsteady simulation setup}

\section{Background of the pump model}

Centrifugal pump model consisting of four blades and four splitters is chosen for the study to operate indirectly as turbine (PAT). The design parameters and hydraulic

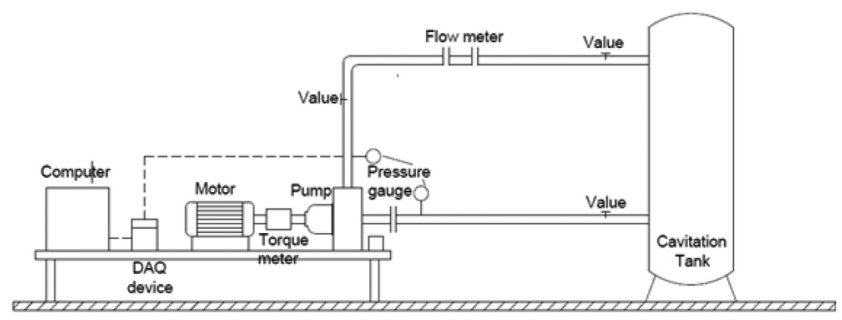

Figure 1. Sketch of the test rig. assessment requirement of PAT are represented in Table 1. Table 2 shows the design schemes of the impeller. The computational flow domain was divided into several components as shown in Figure 3, which were transported into ICEM CFD 14.0 for the mesh generation. Figure 3 shows the view of the mesh topography of the impeller detailing the hub region.

To carry out the PAT simulation, ANSYS CFX 17.5 code was chosen to solve the RANS equation. The initial results obtained from the steady simulation was used to initiate the unsteady calculation. The $k$-omega turbulence model was chosen for the calculation as it came out of the turbulence models, $k-\omega$ and $k-\varepsilon$ and can automatically change between the closed wall limit and major flow direction. Nevertheless choosing the right turbulence model for CFD simulation is essential. The turbulence model $k-\omega$ is a CFD turbulence model for solving two equations of the RANS equation, which helps to predict turbulence. All the other boundary conditions were set for the CFD calculation.

The selected turbulence model for the study is expressed as

$$
\frac{\partial(\rho k)}{\underbrace{\partial t}_{I}}+\underbrace{\frac{\partial}{\partial x_{i}}(\rho \bar{\mu} i \kappa)}_{I I}=\underbrace{\frac{\partial}{\partial x_{i}}\left[\left(\mu+\frac{\mu_{T}}{\sigma_{\kappa}}\right) \nabla \kappa\right]}_{I I I}+\underbrace{P \kappa}_{I V}-\underbrace{\beta * \rho \kappa \omega}_{V}
$$

where,

$$
P_{\kappa}=\left(2 \mu_{T} \frac{\partial \bar{\mu}_{i}}{\partial x_{j}}-\frac{\partial \bar{\mu}_{i}}{\partial x_{j}}-\frac{2}{3} \rho \kappa \frac{\partial \bar{\mu}_{i}}{\partial x_{j}} \delta_{i j}\right) .
$$

Where $\sigma_{\kappa}$ and $\beta^{*}$ are equation constants. Turbulent transport equation frequency $(\omega)$ for $k-\omega$ model can be written as

$$
\begin{aligned}
& \underbrace{\frac{\partial \rho \omega}{\partial t}}_{I}+\underbrace{\frac{\partial}{\partial x_{i}}(\rho \bar{\mu} i \omega)}_{I I}=\underbrace{\frac{\partial}{\partial X_{i}}\left[\left(\mu+\frac{\mu_{T}}{\sigma_{\omega, 1}}\right) \nabla \omega\right]}_{I I I} \\
& \underbrace{\gamma 2\left(2 \rho \frac{\partial \bar{\mu}_{i}}{\partial x_{j}}-\frac{\partial \bar{\mu}_{i}}{\partial x_{j}}-\frac{2}{3} \rho \omega \frac{\partial \bar{\mu}_{i}}{\partial x_{j}} \delta_{i j}\right)}_{I V} \\
& -\underbrace{-\beta_{2} \rho \omega^{2}}_{V I}+\underbrace{2 \frac{\rho}{\sigma_{k}}-\frac{\partial \kappa}{\partial x_{\kappa}}-\frac{\partial \omega}{\partial x_{\kappa}}}_{\underbrace{}_{\omega, 2} \omega} .
\end{aligned}
$$

Momentum is governed by

$$
\frac{\partial \mu_{i}}{\partial t}+u_{j} \frac{\partial \mu_{i}}{\partial x_{j}}=\frac{1}{\rho}\left(\frac{\partial p}{\partial x_{i}}+\frac{\partial T_{i j}}{\partial x_{j}}\right)+f_{t} .
$$



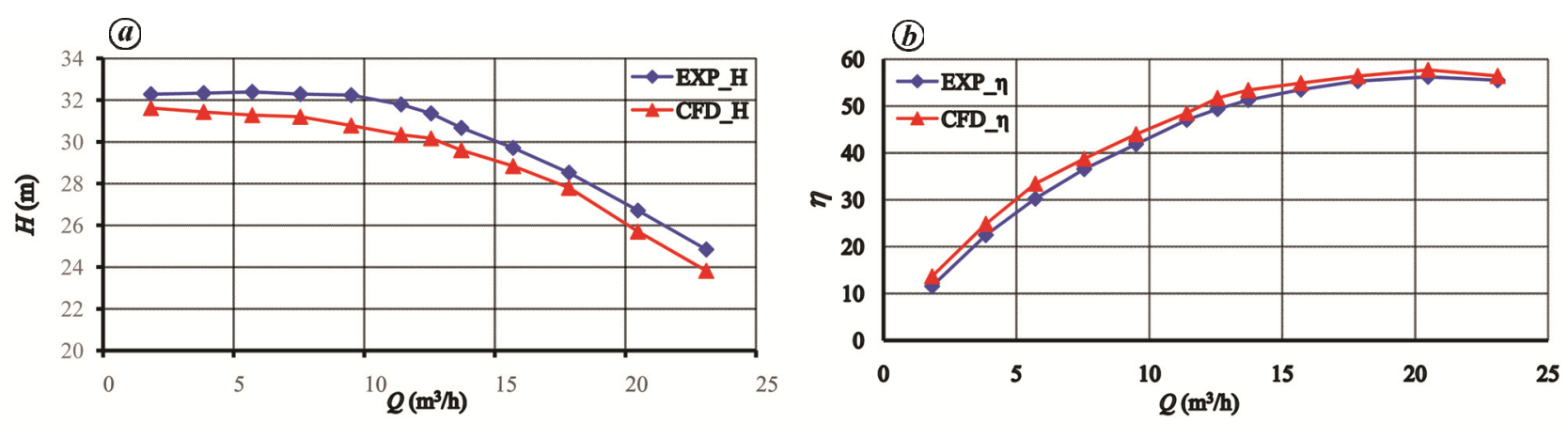

Figure 2. $\boldsymbol{a}$, Head versus flow rate characteristics curve of the selected pump model at $n_{\mathrm{p}}=1400 \mathrm{rpm}$. $\boldsymbol{b}$, Efficiency versus flow rate characteristics curve of the selected pump model at $n_{\mathrm{p}}=1400 \mathrm{rpm}$.

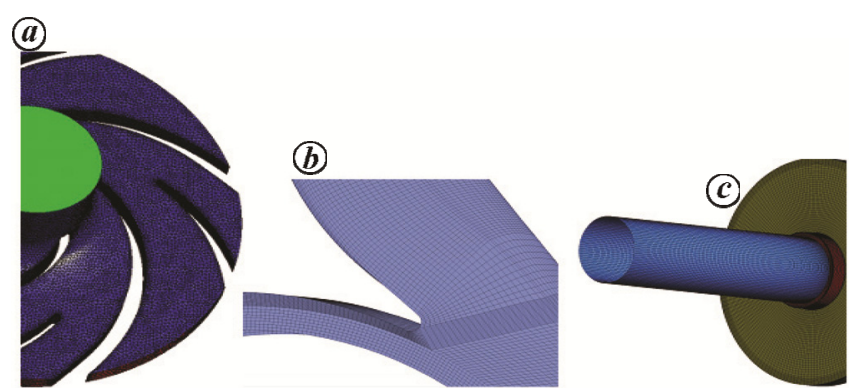

Figure 3. Entire mesh domain of the selected pump under study: $\boldsymbol{a}$, impeller; $\boldsymbol{b}$, volute tongue, $\boldsymbol{c}$, outlet pipe.

Table 1. Parameters of the model centrifugal pump

\begin{tabular}{|c|c|c|c|}
\hline Diameter $D_{1}$ & $50(\mathrm{~mm})$ & Pump flow rate $Q_{\mathrm{d}}$ & $12.5\left(\mathrm{~m}^{3} / \mathrm{h}\right)$ \\
\hline $\begin{array}{c}\text { Outlet impeller } \\
\text { diameter } D_{2}\end{array}$ & $160(\mathrm{~mm})$ & Pump head $H_{\mathrm{d}}$ & $32(\mathrm{~m})$ \\
\hline Impeller width $b_{2}$ & $6(\mathrm{~mm})$ & Efficiency $\eta$ & $56 \%$ \\
\hline Rotational speed $n$ & $1400 \mathrm{r} / \mathrm{min}$ & Specific speed $n_{\mathrm{s}}$ & 47 \\
\hline
\end{tabular}

Table 2. Schematic arrangements of the impeller

\begin{tabular}{lcc}
\hline Long blades & 4 & 4 \\
Splitter blades & - & 4 \\
Splitter Inlet diameter $D_{\mathrm{i}} / \mathrm{mm}$ & - & 104 \\
Blade offset angel $\theta /^{\circ}$ & - & 5 \\
\hline
\end{tabular}

\section{Results discussion}

When a load higher than the design load of a turbine is applied at a constant flow rate, turbine speed decreases. Nevertheless, as this load reduces with constant flow and head, the turbine speed increases ${ }^{17}$. This principle applies in PAT and plays a vital role in determining PAT performance characteristics. With the PAT load decrease, the speed of the PAT attains a maximum value such that no torque can be delivered at this speed, also called runaway speed. The runaway speed of the PAT was realized at flow $\phi=0.03$ with efficiency $\eta=0.53$ as shown in Figure $4 a$. It is observed that as PAT increases with increase in flow rate, the power output also increases. Efficiency, on the other hand, increased with the increase in the flow rate of the impeller until the maximum point was attained at $\phi=1.0$, the best efficiency point $\left(Q_{\mathrm{BEP}}\right)$, after which it began to reduce steadily again as shown in Figure 4 . This means PAT operates better in the overload than the patload condition since lower efficiencies were recorded at lower flow rates. PAT efficiency performs similarly as the power output. It can, therefore, be concluded that the best-operating conditions for the selected PAT could be achieved when it operates at the overload condition and best efficiency point. The dimensionless were calculated using the following formulae.

Power coefficient is expressed as

$$
\pi=\frac{P}{\rho \omega^{3} D^{5}} .
$$

Flow coefficient is expressed as

$$
\phi=\frac{Q}{\omega D^{3}},
$$

where $D$ is the diameter, $Q$ the flow rate, $P$ the power, $n$ the rotation speed, $\pi$ the power coefficient, $\eta$ the efficiency, $\omega$ the angular velocity, $\rho$ the density and $\phi$ is the flow coefficient.

As seen in Figure 5, PAT mode internal flow characteristics were investigated using the head pulsation curve plotted against the time step to find the maximum and minimum head data. Different plots can be observed thus major and minor peaks for the blade and splitter numbers whereas the maximum and minimum points are also shown. The analysis was carried out at the maximum point since the average static pressure and velocity distribution obtained at the highest gridding point was more compared to the lowest gridding point. Static pressure distribution at the higher and lower heads are shown in Figure 5. Pressure was analysed at both time steps 

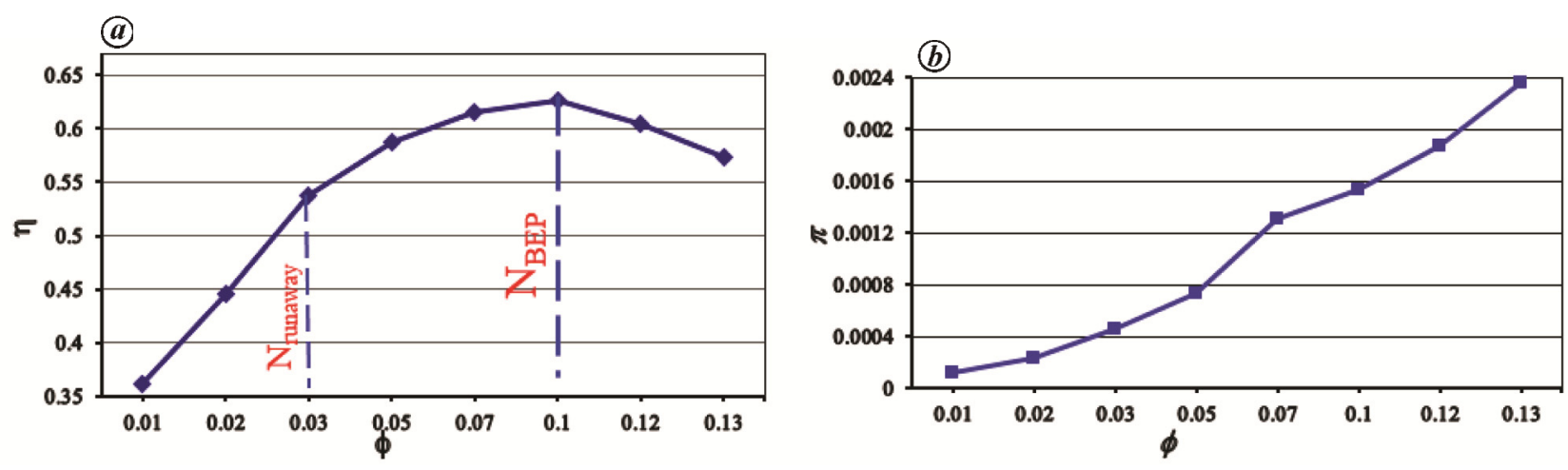

Figure 4. $\boldsymbol{a}$, Efficiency versus flow rate for selected PAT at $N_{t}=1500 \mathrm{rpm}$. $\boldsymbol{b}$, Power versus flow rate for selected PAT at $N_{t}=1500 \mathrm{rpm}$.

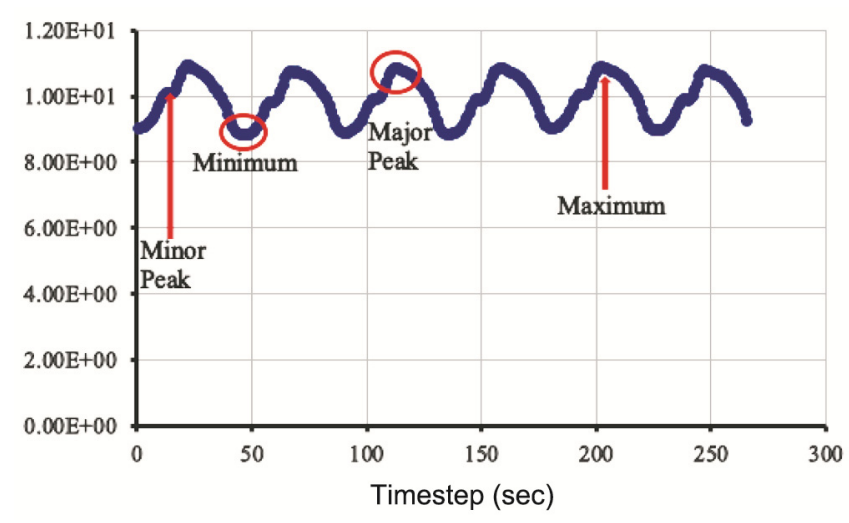

Figure 5. PAT mode head pulsation curve.

and the results show that time steps have an influence on pressure distribution. An increase in the time step increases pressure till it reaches the maximum as shown in Figure 6.

\section{Static pressure distribution for PAT}

Figure 7 displays the graphical pump as turbine pressure distribution at varied flow rate. Pressure variations derived from numerical calculations are obtained in the form of pressure contours. The results revealed that pressure distribution around the blade is asymmetrically disseminated with the pressure side as well as inlet of the rear of the suction side impeller blades recording the maximum pressure. The difference in the flow pattern with the change in flow rate is shown. The results revealed that the pressure distribution in PAT mode operation increases as flow increases. Maximum pressure was recorded at $Q=12.5 \mathrm{~m}^{3} / \mathrm{h}$ where higher pressure was observed at the trailing edge of the blade and the volute tongue region as shown in Figure 7 . However, at flow $Q=15 \mathrm{~m}^{3} / \mathrm{h}$ the highest pressure was recorded around the inlet pipe and tongue reduced as it moved along the flow passage of the impeller blade. The figure shows minimal pressure at the leading edge of the impeller blade of the
PAT. This implies that flow rate can influence PAT performance. It is therefore important to select appropriate flow for PAT operation. In this study, the PAT performed well at flow $Q=12.5 \mathrm{~m}^{3} / \mathrm{h}$. At low flow rates, high recirculation of flow arises in the blade suction part, though movement in the pressure side is smooth. On the other hand, increase in flow and flow parting along the pressure side of the impeller blade occur, which results in the continuous movement of flow in the pressure side. The movement in the suction side of the impeller blade decreases with an increase in flow rate. As kinetic energy flowed from the inlet through to the trailing edge and was delivered to the fluid, the static pressure gradually increased. As seen in Figure 7, due to the conversion of kinetic energy of the fluid to pressure energy, the static pressure further increased due to the increase in section area. For better understanding and explanation on how the fluid acts in the fluid volume, the $3 \mathrm{D}$ static pressure distribution contour of PAT has been provided.

\section{Velocity distribution for PAT}

The movement of velocity in the selected PAT for this study is shown in Figure 8. Results revealed that there exists a steady rise in velocity as the volute tongue region decreases due to decrease in transversal area of the inlet pipe despite the increase in flow rate velocity. The flow moves effectively inside the volute region at a nominal flow rate. At flow $Q=12.5\left(\mathrm{~m}^{3} / \mathrm{h}\right)$ the highest velocity was observed at the tongue region. Nevertheless, the maximum velocity was found at the volute tongue region and the inlet pipe, at each flow condition. The minimum velocity was recorded at the suction as well as the discharge region. Water enters through the suction side and moves along the impeller blades to cause the velocity to increase based on the curving of the blade in the streamwise direction, as the passage way slowly narrows down. Hence, a portion of the work performed by the fluid on the impeller for PAT, is caused by a reaction from the pressure drop. The other part is caused by a change in kinetic energy. The figures in Figure 8 provide better 

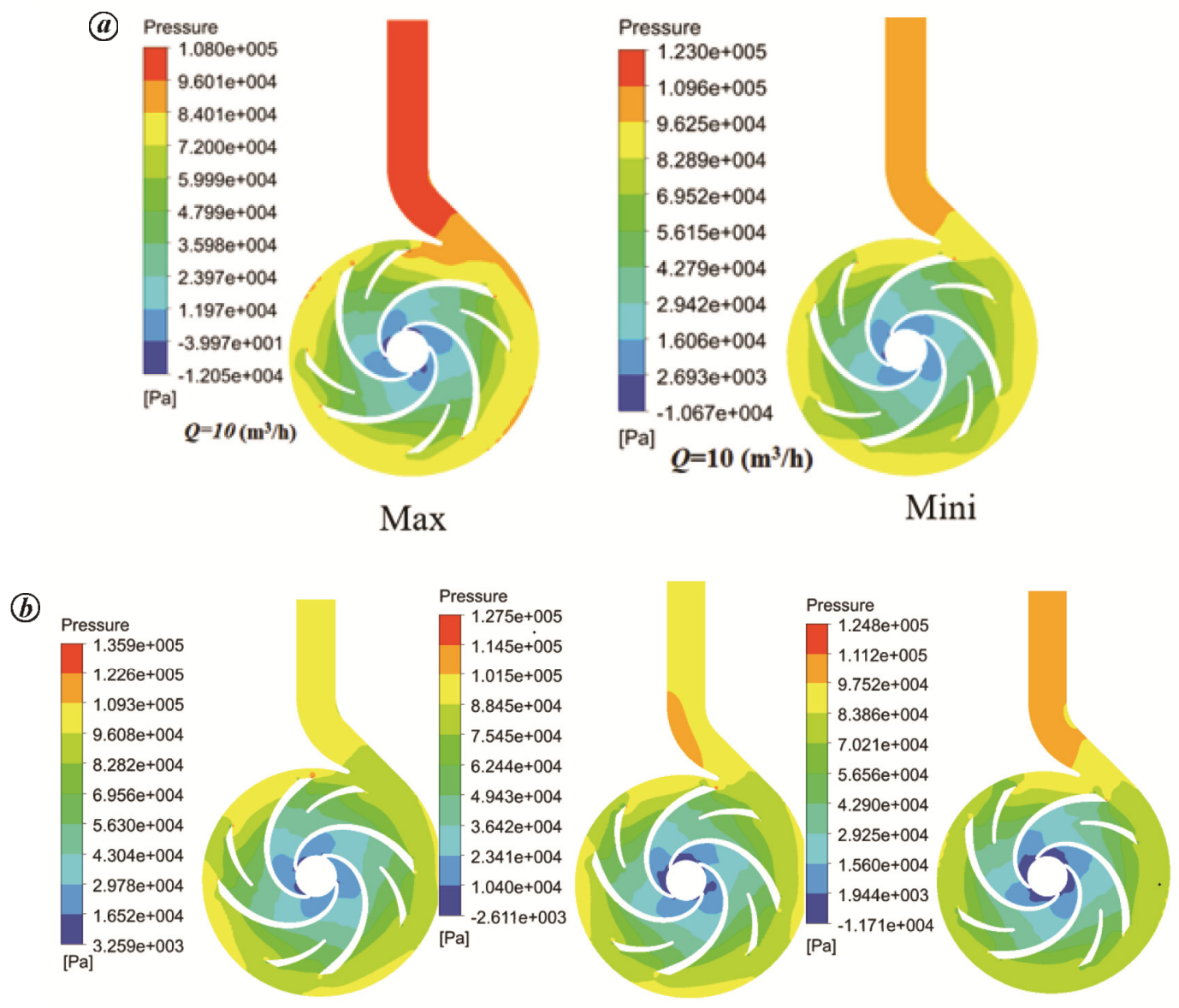

Figure 6. $\boldsymbol{a}$, Distribution of pressure at maximum and minimum of PAT. $\boldsymbol{b}$, Pressure distribution at different time steps of PAT.

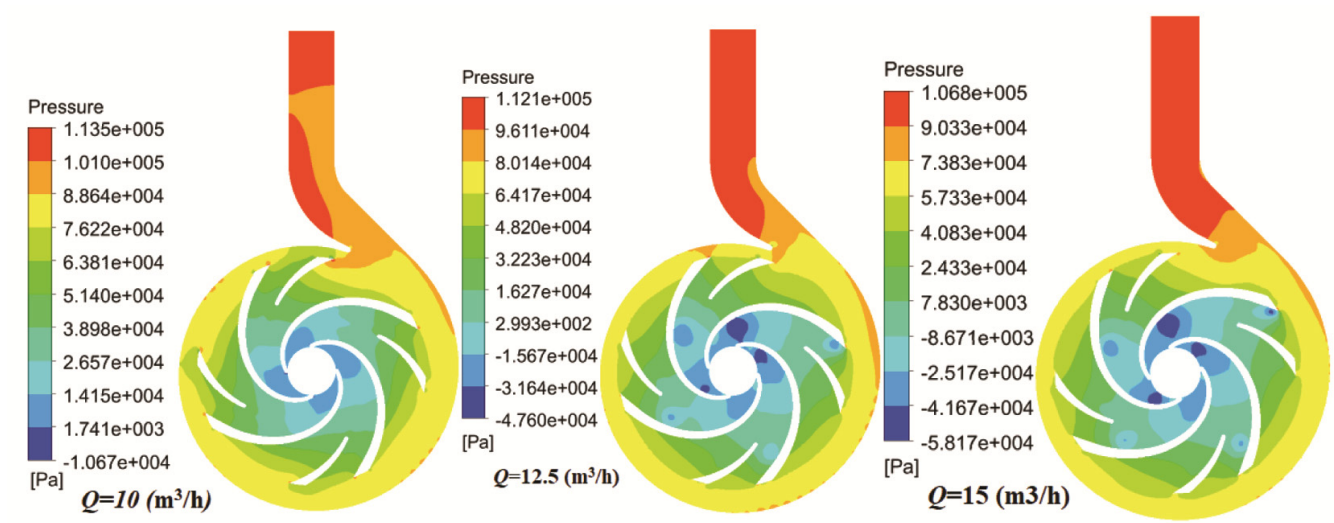

Figure 7. Circulation of pressure in PAT model at dissimilar flow conditions $n=1500 \mathrm{rpm}$.

explanation to the velocity flow results under dissimilar flow conditions.

Figure 9 shows PAT velocity streamline distribution at different flow conditions such as $Q=10\left(\mathrm{~m}^{3} / \mathrm{h}\right), Q=12$ $\left(\mathrm{m}^{3} / \mathrm{h}\right)$ and $Q=15 \mathrm{~m}^{3} / \mathrm{h}$. There is an impact of the velocity flow section on the pressure performance inside the PAT at different flow rates. Velocity streamwise distribution in PAT was the same as the pressure distribution performance. The maximum velocity was recorded at the blade trailing side of the PAT impeller, whereas lowest velocity value was detected at the impeller eye region. However, the velocity steadily rose from the inlet through the pressure side to the trailing edge in radial direction, and this was caused by the asymmetric shape of the volute ${ }^{17}$. The results also revealed that velocity in the entire PAT increased and became unvarying with increase in flow rate. Hence $Q=10 \mathrm{~m}^{3} / \mathrm{h}$ recorded the minimum velocity, with $Q=15 \mathrm{~m}^{3} / \mathrm{h}$ recording the maximum velocity. 


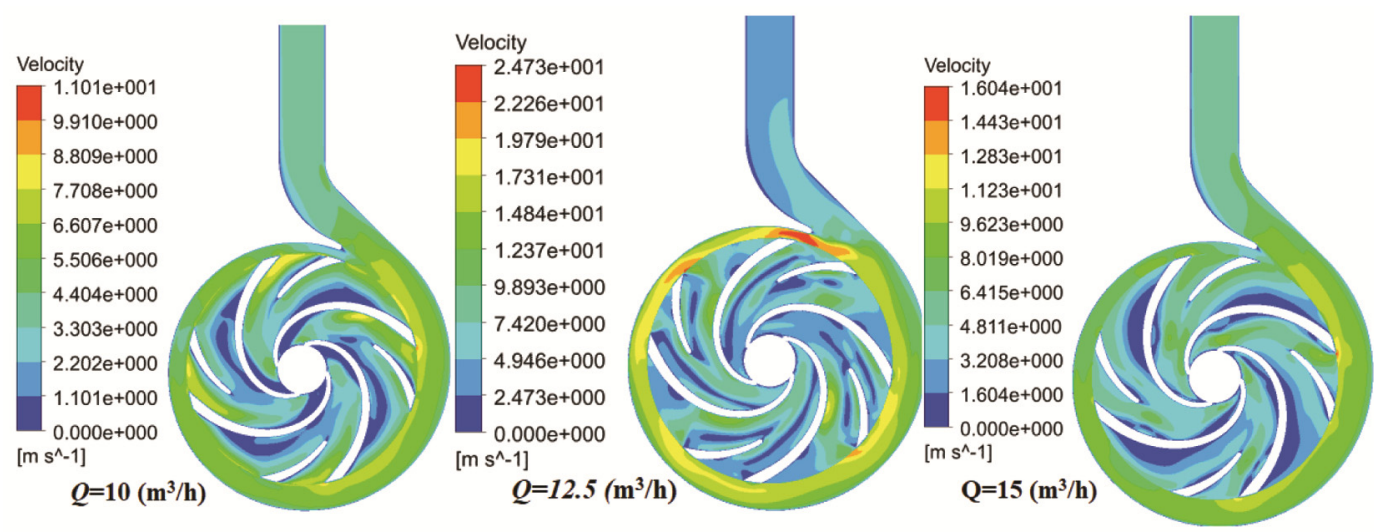

Figure 8. Circulation of velocity in PAT model at dissimilar flow conditions $n=1500 \mathrm{rpm}$.
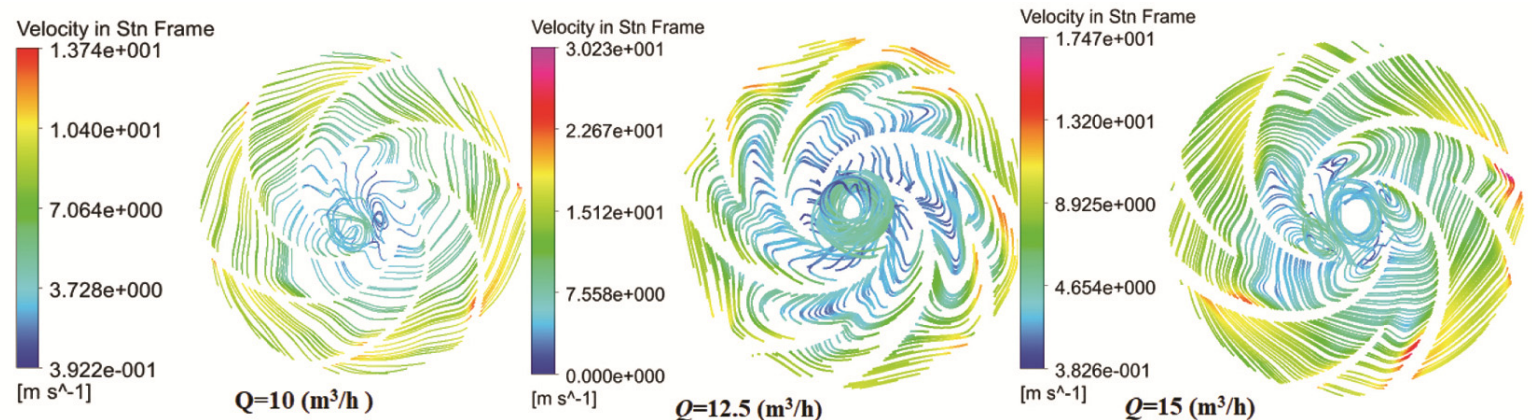

Figure 9. Streamline velocity circulation for PAT at different flow rate $n=1500 \mathrm{rpm}$.

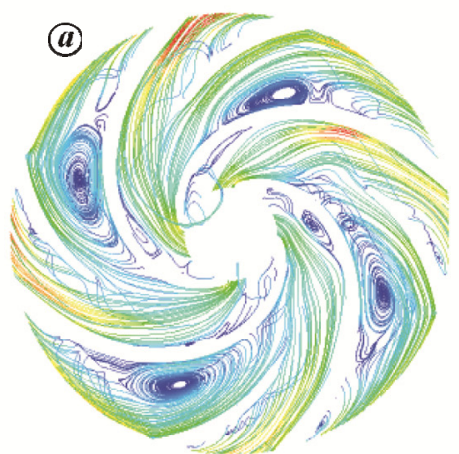

$Q=10\left(\mathrm{~m}^{3} / \mathrm{h}\right)$

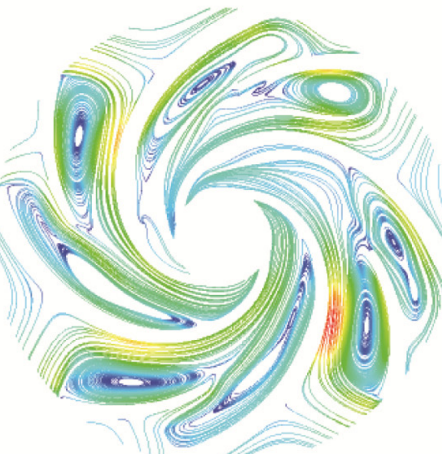

$Q=12.5\left(\mathrm{~m}^{3} / \mathrm{h}\right)$
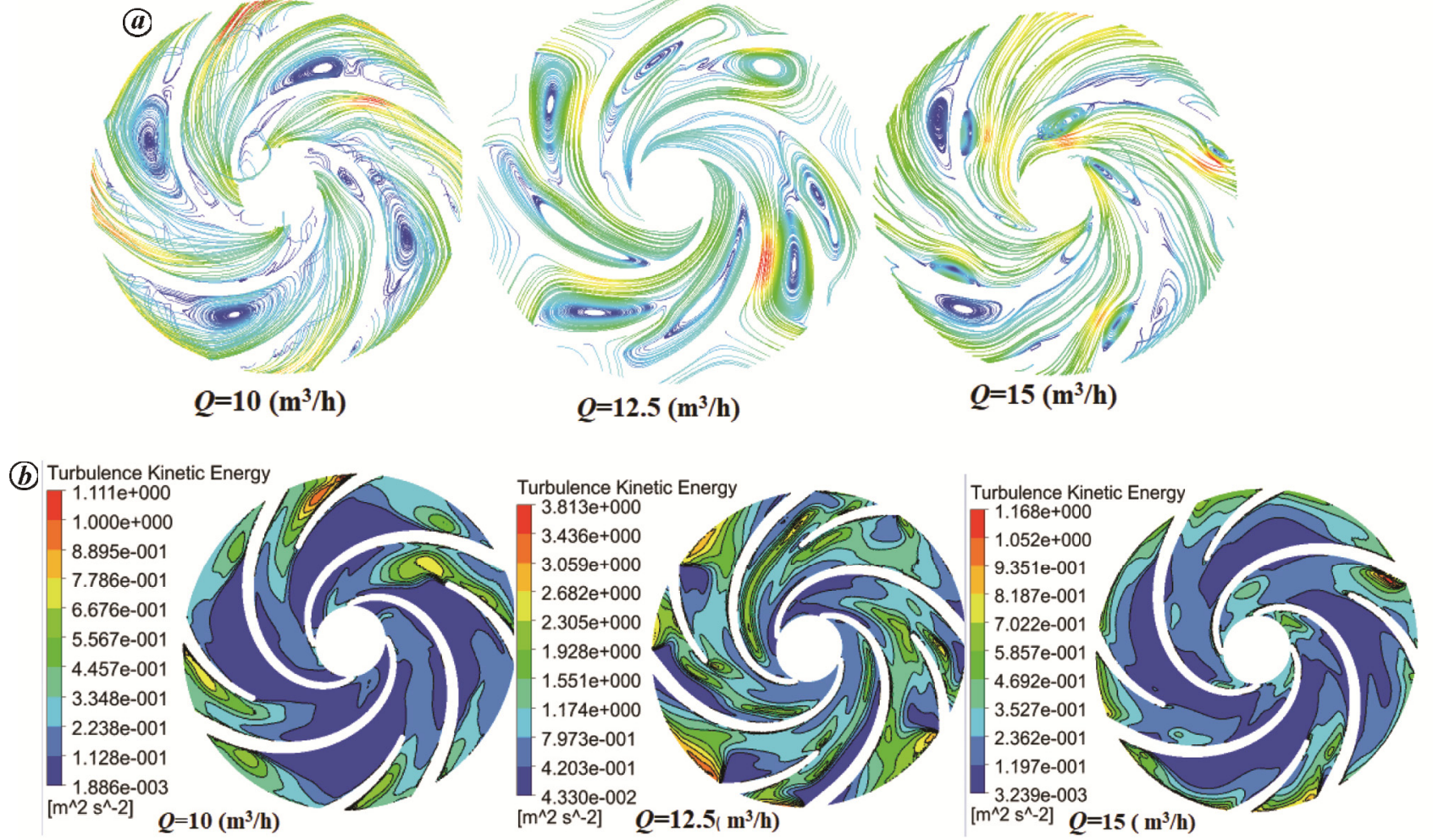

Figure 10. $\boldsymbol{a}$, Streamline vortex circulation of PAT under dissimilar flow rate $n=1500 \mathrm{rpm}$. $\boldsymbol{b}$, Turbulence circulation under dissimilar flow rate $n=1500 \mathrm{rpm}$. 
Figure 10 displays the vortex and turbulence flow circulation in PAT mode with shear stress transport renormalized group $k-\omega$ turbulence model at three different flow conditions. In was observed that there existed higher disparity at the flow passage, which became more uniform with an increase in flow rate; simultaneously, the size of the vortex and quantities decreased. At $Q=10 \mathrm{~m}^{3} / \mathrm{h}$, several vortices of dissimilar dimensions were seen at the flow field. The suction side recorded the maximum vortices at each flow condition as shown in Figure $10 \mathrm{a}$. Suction side in the middle of the impeller flow passage contained more number of and bigger vortices compared to the pressure side. There was uneven distribution of vortices in PAT due to the inlet pipe. The streamline became smooth with minimal vortices recorded at $Q=15 \mathrm{~m}^{3} / \mathrm{h}$. There was asymmetric volute geometry which formed the shortest distance between the impeller outlet and volute wall below the volute tongue region. The faster flow was directed to the volute tongue which caused a vortex below the volute tongue at $Q=10 \mathrm{~m}^{3} / \mathrm{h}$ in Figure $10 a$. The existence of unsteady turbulent flow in PAT showed that the vortex can greatly impact flow parting. The maximum turbulence was observed at the impeller trailing edge at all conditions. Movement of the flow increased turbulence. However as flow rate rose, turbulence gradually decreased as seen in Figure $10 \mathrm{~b}$.

\section{Conclusion}

In this manuscript, the internal flow behaviour of pump as turbine at different flow conditions was analysed. The results can help in PAT selection for constructing a small hydropower site. The results revealed that existence of unsteady turbulent flow in PAT resulted in higher vortex influence on flow parting. Also flow rate has an influence on performance since the change in flow rate increases the velocity and reduces the pressure. As discussed in the results, velocity in the entire PAT increased and became unvarying with increasing flow rate. The deviation was recorded at the design condition by comparing outcomes of the experiment and CFD for head and efficiency. This deviation might be due to the lack of recognition of machine-driven losses by CFD. Therefore numerical results are reliable for PAT selection. PAT BEP shifted near higher discharge with high-efficiency range when the flow rate increased. PAT numerical results showed the existence of rise in flow condition at the site when PAT operated from a lower head to a higher head.

1. Nautiyal, H. and Kumar, A., Reverse running pumps analytical, experimental and computational study: a review. Renew. Sust. Ener. Rev., 2010, 14, 2059-2067.

2. Zhang, J., Adu, D., Fang, Y. and Yin, T., Review on sub-Saharan African small-hydropower situation. Proceedings of the Institution of Civil Engineers - Energy, 2017, jener.17.00025.
3. Agarwal, T., Review of pump as turbine (PAT) for microhydropower. Int. J. Emerg. Technol. Adv. Eng., 2012, 2(11), 163168.

4. Williams, A., Pumps as turbines for low-cost micro hydro power. Renew. Energy, 1996, 9, 1227.

5. Derakhshan, S. and Nourbakhsh, A., Experimental study of characteristic curves of centrifugal pumps working as turbines in different specific speeds. Exp. Therm. Fluid Sci., 2008, 32, 800-807.

6. Amelio, M. and Barbarelli, S., A one-dimensional numerical model for calculating the efficiency of pumps ad turbines for implementation in micro hydropower plants. In ASME 7th Biennial Conference on Engineering System Design and Analysis, 2004, pp. 65-77.

7. Dixon, S. L., Fluid Mechanics and Thermodynamics of Turbomachinery, Elsevier, 2005, 5th edn.

8. Fernandez, J. et al., Performance of a centrifugal pump running in inverse mode. J. Power Energ., 2004, 4, 265-271.

9. Singh, P. and Nestmann, F., An optimisation routine on a prediction and selection model for the turbine operation of centrifugal pumps. Exp. Therm. Fluid Sci., 2010, 34(2), 152-164.

10. Derakhshan, S., Nourbakhsh, A. and Mohammadi, B., Efficiency improvement of centrifugal reverse pumps. J. Fluids Eng., 2009, 131(2), 9 .

11. Joshi, S., Holloway, A. G. L. and Chang, L., Selecting a high specific speed pump for low head hydroelectric power generation. IEEE Trans., 2005, 75, 816-826.

12. Nautiyal, H., Varun Kumar, A. and Yadav, S., Experimental investigation of centrifugal pump working as turbine for small hydropower systems. Energy Sci. Technol., 2011, I(1), 79-86.

13. Barrio, R., Fernandez, J., Par Rondo, J. and Blanco, E., Performance prediction of a centrifugal pump working in direct and reverse mode using computational fluid dynamics. In Proceedings of the International Conference on Renewable Energies and Power Quality, Granada, Spain, 2010, pp. 1-5.

14. Shukla, A., Performance evaluation of pump as turbine [M Tech thesis], Indian Institute of Technology Roorkee, 2008.

15. Rawal, S. and Kshirsagar, J. T., Numerical simulation on a pump operating in a turbine mode. In Proceedings of the Twenty-Third International Pump Users' Symposium, Texas, Houston, 2007, pp. 21-27.

16. Morros, C. S., Oro, J. M. F. and Diaz, K. M. A., Numerical modelling and flow analysis of a centrifugal pump running as a turbine: unsteady flow structures and its effects on the global performance. Int. J. Numer. Methods Fluids, 2011, 65(5), 542-562.

17. Carravetta, A., Fecarotta, O., Martino, R. and Antipodi, L., PAT efficiency variation with design parameters. Proc. Eng., 2014, 70, 285-291.

18. Zhang, J. et al., Numerical investigation of transient vortices and turbulent flow behaviour in centrifugal pump operating in reverse mode as turbine. Mater. Sci. Energy Technol., 2018; doi:doi.org/ 10.1016/j.mset.2018.12.002

19. Fan Zhang et al., Transient flow characterization in energy conversion of a side channel pump under different blade suction angles. Energy, 2018, 161, 635-648.

20. Fan Zhang et al., Effect of blade profile for suction side on the performance of a side channel pump. Proc. Ins. Mech. Eng., A-J. Pow. Energy, 2016, 230(6), 586-597.

ACKNOWLEDGEMENT. We thank the National Natural Science Foundation of China for their financial support with Grant No. 51762042 .

Received 30 January 2019; revised accepted 25 February 2019

doi: $10.18520 / \mathrm{cs} / \mathrm{v} 117 / \mathrm{i} 1 / 57-63$ 